\title{
Interval cancers after negative immunochemical test compared to screen and non-responders' detected cancers in Slovenian colorectal cancer screening programme
}

\author{
Dominika Novak Mlakar ${ }^{1,2}$, Tatjana Kofol Bric ${ }^{1}$, Ana Lucija Škrjanec ${ }^{1}$, Mateja Krajc ${ }^{3}$ \\ ${ }^{1}$ National Institute of Public Health, Cancer Screening, Ljubljana, Slovenia \\ ${ }^{2}$ University of Ljubljana, Faculty of Medicine, Ljubljana, Slovenia \\ ${ }^{3}$ Institute of Oncology Ljubljana, Cancer Genetic Clinic, Ljubljana, Slovenia
}

Radiol Oncol 2018; 52(4): 413-421.

Received 17 April 2018

Accepted 20 May 2018

Correspondence to: Assist. Prof. Mateja Krajc, M.D., Ph.D., Institute of Oncology Ljubljana, Zaloška cesta 2, SI-1000 Ljubljana, Slovenia. Phone: + 38615879 635; Fax: + 38615879 400; E-mail: mkrajc@onko-i.si

Disclosure: No potential conflicts of interest were disclosed.

Background. We assessed the incidence and characteristics of interval cancers after faecal immunochemical occult blood test and calculated the test sensitivity in Slovenian colorectal cancer screening programme.

Patients and methods. The analysis included the population aged between 50 to 69 years, which was invited for screening between April 2011 and December 2012. The persons were followed-up until the next foreseen invitation, in average for 2 years. The data on interval cancers and cancers in non-responders were obtained from cancer registry. Gender, age, years of schooling, the cancer site and stage were compared among three observed groups. We used the proportional incidence method to calculate the screening test sensitivity.

Results. Among 502,488 persons invited for screening, 493 cancers were detected after positive screening test, 79 interval cancers after negative faecal immunochemical test and 395 in non-responders. The proportion of interval cancers was 13.8\%. Among the three observed groups cancers were more frequent in men $(p=0.009)$ and in persons aged $60+$ years $(p<0.001)$. Comparing screen detected and cancers in non-responders with interval cancers more interval cancers were detected in persons with 10 years of schooling or more $(p=0.029$ and $p=0.001)$, in stage III $(p=0.027)$ and IV $(p<0.001)$, and in right hemicolon $(p<0.001)$. Interval cancers were more frequently in stage I than non-responders cancers $(p=0.004)$. Test sensitivity of faecal immunochemical test was $88.45 \%$.

Conclusions. Interval cancers in Slovenian screening programme were detected in expected proportions as in similar programmes. Test sensitivity was among the highest when compared to similar programmes and was accomplished using test kit for two stool samples.

Key words: colorectal cancer screening; faecal immunochemical test; interval cancer; screen detected cancer; cancer in non-responders; test sensitivity

\section{Introduction}

Colorectal cancer is a major public health problem in developed world. In 2012, it was the third most common cancer in the world with 1.4 million new cases and was responsible for $8.5 \%$ of all cancer-related deaths. ${ }^{1}$ In Slovenia, the average incidence of colorectal cancer in the period 2009-2013 was 1,569 cases per year. Incidence rate was higher in men $(92.4 / 100,000)$ than in women $(60.8 / 100,000)$. It was the third most common cancer and was responsible for $13.6 \%$ of all cancer-related deaths. ${ }^{2}$

In 2003, Council of the European Union proposed to member states to introduce a population based organised screening programme for three cancer sites (colorectum, cervix and breast) and 
thus reduce the burden caused by the disease. ${ }^{3}$ The implementation of organized population colorectal cancer screening proved to reduce the mortality successfully. Using a guaiac screening test, the colorectal cancer mortality in apparently healthy population with moderate risk was reduced by 15.0 to $33.0 \%$.

European Guidelines for Quality Assurance in Colorectal Cancer Screening and Diagnosis recommend faecal occult blood test as a screening test.? It does not have a diagnostic characteristic in detecting colorectal neoplasms, since any intestinal bleeding can result in positive test. ${ }^{7,8}$ Therefore, colonoscopy is recommended as a diagnostic method for detecting premalignant and early stage cancers after positive screening tests. ${ }^{7,8}$

Next to positive effects, screening also has some negative effects for the screenees if the test result is false positive or false negative. ${ }^{3} \mathrm{~A}$ good screening test should have a small number of false positive results and, even more important, a small number of false negative results in those who already have cancer. ${ }^{4}$ Experience show that in colorectal cancer screening programmes the so-called interval cancers are detected after negative screening tests. ${ }^{7,9}$

As published definitions of interval cancer differ, it is difficult to compare results of different studies. A group of experts has been working on the recommended definitions of interval cancer after a negative screening test and after colonoscopy or any other comparable diagnostic test. ${ }^{7}$ International working group has developed a nomenclature for the definition and description of interval cancers in colorectal cancer screening programme. ${ }^{10}$

Interval cancer rarely appears in previously healthy intestinal mucosa in the period between two screening tests. Most probably screening test misses some neoplasms. ${ }^{10,11}$ The proportion of interval cancers is an important performance indicator of the screening programme and it reflects the test sensitivity (false negative) as well as the incidence of newly detected cancers which were not present during the screening. ${ }^{7,11}$ According to the European guidelines, the data on the incidence of interval cancers should be collected and reported, which enables the monitoring of programme effectiveness. ${ }^{7}$

More frequent incidence of colorectal cancer in men, in older people and in persons with lower socio-economic status is reflected in screen detected cancers, whereas there is no uniform conclusion regarding the characteristics of interval cancer incidence. These findings can contribute to the adaptation of screening programmes to groups at higher risk for interval cancer. . $^{1,2,11-13}$
In 2008, a colorectal cancer screening pilot project confirmed the feasibility of planned procedures as well as the effectiveness of cancer detection in early stages with the organized population screening in Slovenia. ${ }^{14}$ In April 2009, a national colorectal cancer screening programme named Programme Svit, started in Slovenia. ${ }^{15}$ Each programme screening round lasts for two years. In first three screening rounds, the target group included men and women aged 50 to 69 years. Since 2015, the upper age limit of target population is set to 74 years..$^{15}$

In our study, we analysed the data on interval cancers after negative result of faecal immunochemical test in Slovenian colorectal cancer screening programme. The data on interval cancers are important for the assessment of screening test sensitivity. The aim of the analysis was to assess the incidence and characteristics of interval cancers in colorectal cancer screening programme as well as the characteristics of the affected population, and to compare them to screen detected cancers and cancers in non-responders. Data on interval cancers after follow-up colonoscopy without detected cancer were not available yet in Slovenian colorectal cancer screening programme and were not the topic of this article.

\section{Patients and methods}

\section{Population and research design}

The study population represent the total target population of the colorectal cancer screening programme in Slovenia at the time being. People were invited in the second programme screening round, which lasted from April 2011 until December 2012. We divided the cohort into three groups, namely non-responders which did not respond to the invitation or returned the test kit, test positive population, and test negative population. We followed-up all groups for two years and extracted records of their new colorectal cancers from Cancer Registry of Republic of Slovenia. For the study purpose, we obtained the data on cancers detected during the follow-up period until the end of incidence year 2014 from cancer registry in 2017. This ensured the time, in which the cancer registry was able to identify cancers detected within and out of the screening programme and to obtain all the necessary data. Demographic data of the followed-up population (name, family name, date of birth, address of residence) were obtained from Central Population Registry. To calculate the follow-up time in personyears we obtained the data on deceased persons in 
all three groups from Causes of Deaths Database of the National Institute of Public Health.

The data on the educational attainment of cancer cases patient were obtained from the Republic of Slovenia Statistical Office database. Educational attainment was converted into two groups, whereby less and up to including 9 years of schooling represents primary school, 10 or more years of schooling represents vocational or secondary school, higher educational level and all subsequent levels.

The data on faecal immunochemical tests, colonoscopies results and the characteristics of screen detected cancers were obtained from national central colorectal cancer screening registry. The data on interval and non-responders' cancer cases were obtained from the cancer registry.

\section{The invitation and screening test}

The population, aged 50 to 69 years, which was invited into the second programme screening round, received the invitation to participate in the screening programme. Persons, who have decided to participate and have returned the signed consent, received test kits for two stool samples unless they had self-reported permanent and temporary exclusion criteria. Exclusion criteria for the participation in screening programme are described in Slovene guidelines for colorectal cancer screening quality assurance. ${ }^{16}$

Permanent exclusion criteria group included persons with (i) chronic inflammatory bowel disease; (ii) adenoma; and (iii) colorectal cancer diagnosed prior to entering the screening programme.

Persons, who have undergone a colonoscopy procedure in the past three years and the above mentioned pathology was not detected, were included in the group with temporary exclusion criteria and were again invited for screening in the next programme screening round.

At first, a qualitative immunochemical test for faecal occult blood (MagStream HT, Fujirebio, Japan) was used as a screening test. After the new public tender for screening test supply the quantitative immunochemical test (OC - Sensor, Eiken Chemical Co, Tokyo, Japan) has been used since 2011. The latter test enables the measurement of haemoglobin quantity in the stool sample, which was not possible with the qualitative test indicating only positive or negative result. We used two stool samples in each test kit from the start of the screening programme. Positive result in quantitative test meant that haemoglobin value in one of two stool samples was higher than $20 \mu \mathrm{g} \mathrm{Hb} / \mathrm{g}$ of stool or higher than $100 \mathrm{ng} \mathrm{Hb} / \mathrm{mL}$ of buffer. Among the study population, which has performed a screening test, $23.9 \%$ of persons had a qualitative test and $76.1 \%$ performed a quantitative test.

\section{Screening test results}

Persons with negative screening test received the test results with an explanation that specimen samples did not exceed the haemoglobin cut-off value and that they do not need additional tests, but have to be aware of new symptoms.

Persons with positive test results were referred to the colonoscopy. Colonoscopies were performed in average in 42 days after the stool samples analysis. In some cases, the colonoscopy date was postponed due to patient's health condition or his/her wish to undergo the colonoscopy in a particular colonoscopy centre, where waiting period was longer.

\section{Colorectal cancer cases classification according to detection mode}

In order to identify the cancer cases among the study population we linked individual data with cancer registry data based on the uniform identification number. For all three cancer detection mode groups (screen detected, interval cancers after negative faecal screening tests, non-responders' cancers), we used the cancer registry to obtain the data on cancer TNM stage, cancer site, histological type and diagnosis date.

Cancer cases, who had negative faecal screening test and a colorectal cancer diagnosis in cancer registry, were classified as patients with interval cancer. Cancer diagnosis date was between the date of stool sample testing and the date of the next foreseen screening or before new invitation to the next screening round.

Cancer cases, who had positive screening test, with a lesion detected and histologically confirmed as cancer were classified as screen detected cancer. In screen detected cancer group two subgroups of patients were followed. In the first subgroup, colonoscopy was performed within the screening programme. In the second subgroup, cancer was detected with colonoscopy outside the screening programme after positive immunochemical test in the screening programme and cancer diagnosis date registered in cancer registry was inside six months from positive test in the screening.

Cancer cases, who did not respond to the invitation or did not supply the stool samples, were classified as non-responder cancer patients. They had 


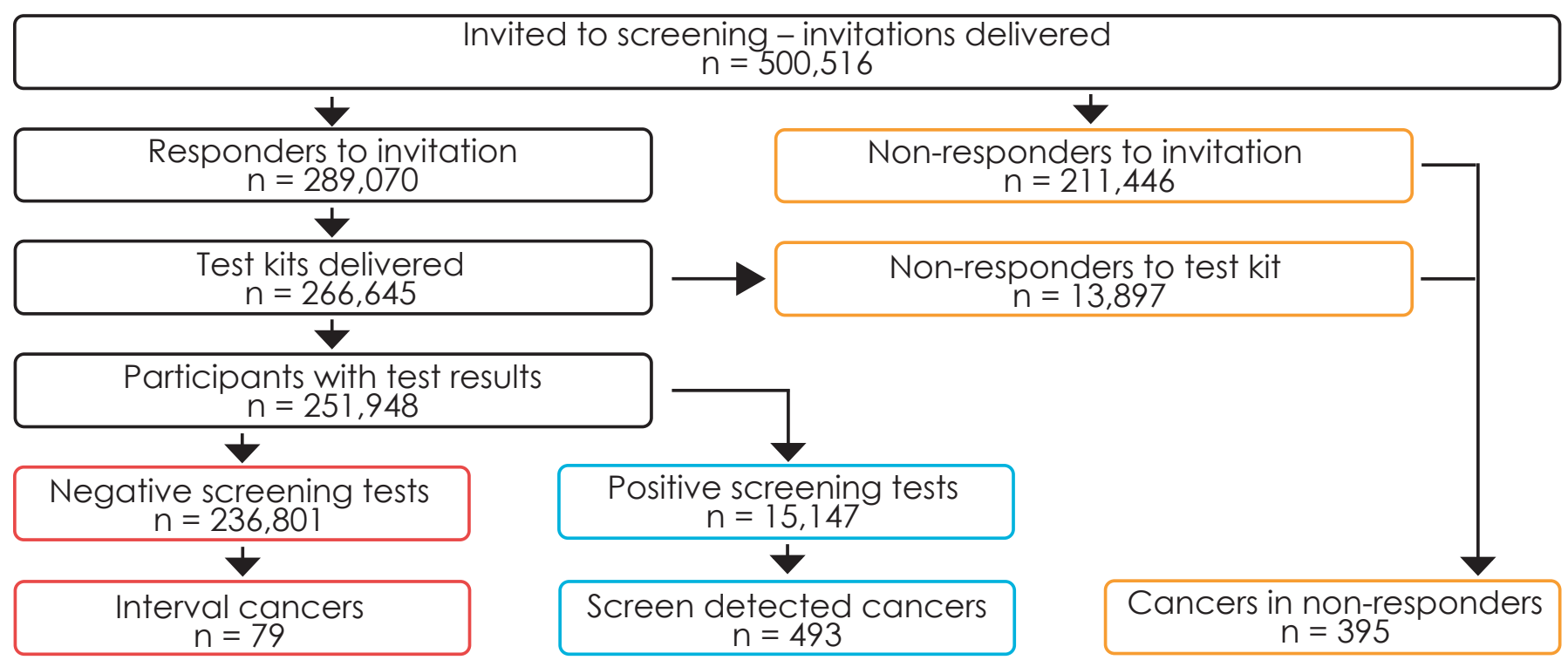

FIGURE 1. Colorectal cancer screening flowchart, including screen detected, interval and non-responders' cancers.

colorectal cancer diagnosis in cancer registry. The diagnosis date was between the date of the sent invitation or test kit and the date of invitation to the next screening round.

Cases, which were detected between caecum and splenic flexure (according to ICD-10, codes C180-C185, except C181), were classified as proximal colorectal cancers. Cases, detected in and between descendent colon and rectum (ICD-10 codes C186-C20), were classified as cancers in the left (distal) part of the colorectum.

The analysis included cancer cases with histological diagnosis according to International classification of diseases for oncology with codes 8010/3 to $8580 / 3 .{ }^{17}$ The following neoplasms were also detected in colorectum; but were not included in the analysis: neoplasms without microscopic confirmation, lymphomas, 8124/3-Cloacogenic carcinoma, 8240/3-Carcinoid tumour, NOS, 8249/3-Atypical carcinoid tumour and 8936/3-Gastrointestinal stromal sarcoma.

\section{Ethical aspects of the research}

Republic of Slovenia National Medical Ethics Committee passed a signed consent for the research at the sessions on 16 $6^{\text {th }}$ April 2013 (Nr. 129/04/13) and $14^{\text {th }}$ May 2013 (Nr. 166/05/13).

\section{Statistical analysis}

We used Pearson Chi-Square Test to calculate statistically significant differences among groups of patients with screen detected cancers, interval cancers and non-responder cancers as well as the characteristics of detected cancers.

The proportion of interval cancers after negative screening test was calculated by the division of the number of interval cancers with the sum of interval and screen detected cancers. To assess the screening test sensitivity, we used the proportional incidence method. ${ }^{18-20}$ Screening test sensitivity was calculated using the following equation: $\left\{1-\left(\left(\alpha * \mathrm{I}_{\text {screen }}\right) /\left(\mathrm{I}_{\text {under }}-(1-\alpha) * \mathrm{I}_{\text {non-screen }}\right)\right)\right\} * 100$. Cancer incidence was expressed at 100,000 personyears. ${ }^{18-20}$ The $a$ in the formula represents the proportion of screened persons, $I_{\text {screen }}$ represents the incidence of interval cancers detected after the negative screening test, $I_{\text {under }}$ represents the incidence of colorectal cancers in the population aged 50 to 69 years prior to the introduction of the screening programme, and $I_{\text {non-screen }}$ represents the incidence of non-responder colorectal cancers. The statistical significance level was set to $p<0.05$. For data analysis, we used SPSS statistical programme package, version 21.0 for Windows.

\section{Results}

From April 2011 to December 2012, we mailed 502,488 invitations for participation in the screening to target population individuals aged 50 to 69 years, out of which $49.7 \%$ were men and $50.3 \%$ were women. $12.7 \%$ of them were newly invited to the screening programme and $87.3 \%$ were invited 
TABLE 1. Selected characteristics of colorectal cancer patients and their disease

\begin{tabular}{|c|c|c|c|c|c|c|c|c|c|c|}
\hline & & \multicolumn{2}{|c|}{$\begin{array}{l}\text { Screen detected } \\
\text { cancer (SDC) }\end{array}$} & \multicolumn{2}{|c|}{ Interval cancer (IC) } & \multicolumn{2}{|c|}{$\begin{array}{l}\text { Non-responders } \\
\text { cancer (NRC) }\end{array}$} & \multirow{2}{*}{$\begin{array}{l}\text { p value } \\
\text { (SDC vs } \\
\text { IC) }\end{array}$} & \multirow{2}{*}{$\begin{array}{l}\text { p value } \\
\text { (IC vs } \\
\text { NRC) }\end{array}$} & \multirow{2}{*}{$\begin{array}{l}p \text { value } \\
\text { (SDC vs } \\
\text { NRC) }\end{array}$} \\
\hline & & Number & $\%$ & Number & $\%$ & Number & $\%$ & & & \\
\hline Cancer total & & 493 & & 79 & & 395 & & & & \\
\hline \multirow{4}{*}{ Age } & $50-54$ & 89 & 18.1 & 12 & 15.2 & 73 & 18.5 & \multirow{4}{*}{0.101} & \multirow{4}{*}{0.323} & \multirow{4}{*}{0.119} \\
\hline & $55-59$ & 93 & 18.9 & 10 & 12.7 & 63 & 15.9 & & & \\
\hline & $60-64$ & 182 & 36.9 & 26 & 32.9 & 145 & 36.7 & & & \\
\hline & $65 \leq$ & 129 & 26.2 & 31 & 39.2 & 114 & 28.9 & & & \\
\hline \multirow{2}{*}{ Gender } & Male & 308 & 62.5 & 50 & 63.3 & 271 & 68.6 & \multirow{2}{*}{0.889} & \multirow{2}{*}{0.356} & \multirow{2}{*}{0.057} \\
\hline & Female & 185 & 37.5 & 29 & 36.7 & 124 & 31.4 & & & \\
\hline \multirow{3}{*}{ Years of schooling } & $\leq 9$ years & 146 & 29.6 & 14 & 17.7 & 160 & 40.5 & \multirow{3}{*}{0.029} & \multirow{3}{*}{$<0.001$} & \multirow{3}{*}{0.001} \\
\hline & $\geq 10$ years & 342 & 69.4 & 64 & 81.0 & 230 & 58.2 & & & \\
\hline & Unknown & 5 & 1.0 & 1 & 1.3 & 5 & 1.3 & & & \\
\hline \multirow{2}{*}{ Cancer site } & Distal colon & 380 & 77.1 & 39 & 49.4 & 301 & 76.2 & \multirow{2}{*}{$<0.001$} & \multirow{2}{*}{$<0.001$} & \multirow{2}{*}{0.759} \\
\hline & Proximal colon & 113 & 22.9 & 40 & 50.6 & 94 & 23.8 & & & \\
\hline \multirow{5}{*}{ TNM stage } & । & 251 & 50.9 & 16 & 20.3 & 32 & 8.1 & \multirow{5}{*}{$<0.001$} & \multirow{5}{*}{0.023} & \multirow{5}{*}{$<0.001$} \\
\hline & $\|$ & 99 & 20.1 & 17 & 21.5 & 87 & 22.0 & & & \\
\hline & III & 104 & 21.1 & 25 & 31.6 & 124 & 31.4 & & & \\
\hline & IV & 39 & 7.9 & 19 & 24.1 & 132 & 33.4 & & & \\
\hline & Unknown & 0 & & 2 & 2.5 & 20 & 5.1 & & & \\
\hline
\end{tabular}

for the second time. 500,516 (99.6\%) invitations were delivered. As many as $289,070(57.8 \%)$ persons have responded to the invitations (Figure 1). Due to the exclusion criteria, 22,425 (7.7\%) persons were not sent a test kit. Test kits for two stool samples were mailed to 266,645 (92.2\%) persons who were eligible for screening. As many as 252,653 persons returned the stool samples. Among people with delivered invitation, without the ones with exclusions criteria, $52.8 \%$ were screened. Among the persons with adequate stool samples, 236,801 $(94.0 \%)$ had negative screening test. Among the persons with positive screening test $(15,147$ or $6.0 \%), 92.2 \%$ of them had undergone a colonoscopy in one of the colonoscopy centres appointed by the screening organization.

Table 1 shows the characteristics of study population and detected cancers in case of interval, screen detected and non-responders' cancers. Among 502,488 persons invited for screening, 493 cancers were detected after positive screening test followed by colonoscopy, 79 interval cancers after negative faecal immunochemical test and 395 in non-responders.

Out of 493 screen detected cancer patients, 467 had colonoscopy in one of the appointed centres and 26 outside the screening program. The analy- sis showed that $88(17.8 \%)$ screen detected cancer cases were diagnosed because of the positive result of the second stool sample, whereas the first stool sample test result was negative. Among nonresponders with diagnosed cancer, 374 persons did not respond to the invitation into the screening programme, while 21 persons responded to the invitation but did not return the test kit.

Among the persons with interval cancer, 57 were provided with a quantitative faecal immunochemical test, which enables the measurement of haemoglobin value in the stool sample. In 43 (75.4\%) study persons with interval cancer after the negative quantitative test, the haemoglobin value was between 0.0 and $50 \mathrm{ng} \mathrm{Hb} / \mathrm{mL}$ of buffer, among them there was no trace of haemoglobin ( $0.0 \mathrm{ng} \mathrm{Hb} / \mathrm{mL}$ buffer) in none of the stool samples in 14 persons.

Among the persons with positive test result, the cancer was significantly more often detected in men $(p=0.009)$ and in persons aged $60+$ years $(p<0.001)$. The results were similar in interval cancers after the negative screening test and in nonresponders' cancers, where there were also significantly more cancer cases in men $(p=0.001$ and $\mathrm{p}<0.001)$ and in persons aged $60+$ years $(\mathrm{p}<0.001$ and $\mathrm{p}<0.001$ ). 
FIT test sensitivity $=88.45 \%=$

$$
\begin{aligned}
& =\left\{1-\frac{a \times I_{\text {screen }}}{I_{\text {under }}-(1-a) \times I_{\text {non-screen }}}\right\} \times 100= \\
& =\left\{1-\frac{0.528 \times 16.77}{118.96-(1-0.528) \times 89.68}\right\} \times 100
\end{aligned}
$$

FIGURE 2. Test sensitivity adjusted for selection bias according to the proportional incidence method formula.

In the comparison between interval cancers and screen detected cancers, there were no differences in cancer incidence according to gender and age groups. Among the interval cancer patients, there were significantly more persons, who had 10 years or more of schooling. In screen detected cancer patients, there were more persons with less than 10 years of schooling $(p=0.029)$. Screen detected cancers were significantly more frequent in the left part of the colon, while interval cancers were more frequent in the right part of the colon $(\mathrm{p}<0.001)$. In screen detected cases more cancers were in stage I $(p<0.001)$, whereas in interval cancers more were in stage III $(p=0.027)$ and stage IV $(p<0.001)$.

In the comparison between interval cancers and non-responders' cancers, there was no difference in cancer incidence according to gender and age groups. Among the interval cancer patients, more persons had 10 years of schooling or more, while among the non-responder cancer patients there were more persons with less than 10 years of schooling $(p=0.001)$. Non-responders' cancers were more frequent in the left part of the colon, while interval cancers were more frequent in the right part of the colon $(\mathrm{p}<0.001)$. In interval cancers compared to non-responders' cancers more were detected in stage I $(p=0.004)$.

In the comparison between screen detected cancers and non-responders' cancers, there was no difference according to gender or age groups. Among the non-responder cancer patients, there were more persons with less than 10 years of schooling, compared to screen detected cancer patients, where there were more persons with 10 years of schooling or more $(p=0.001)$. Cancers in both groups were more frequent in the left part of the colon, but there was no significant difference in comparison with the right part of the colon. In screen detected cancers more were in stage I $(p<0.001)$, whereas more non-responder cancers were in stage II $(p=0.016)$ and IV $(\mathrm{p}<0.001)$.

The proportion of interval cancers among cancers detected in second screening round was $13.8 \%$, which means 79 interval cancers after the nega- tive screening test according to the sum of screen detected and interval cancers $(493+79)$. The test sensitivity of faecal immunochemical test used for colorectal cancer screening was $88.45 \%$ (Figure 2).

\section{Discussion}

The analysis represents the first evaluation of the Slovenian colorectal cancer screening programme focused on assessing the incidence and the characteristics of interval cancers after the negative faecal immunochemical screening test, the characteristics of patients with interval cancer, and the comparison of interval cancers with screen detected cancers and cancers in non-responders.

The proportion of interval cancers detected after negative faecal immunochemical screening test was $13.8 \%$. It has somewhat differed in published studies possibly due to different types of immunochemical tests used, different cut-off values set for test positivity, and the number of required stool samples. In Slovenia, two stool samples are required. In research by Zorzi et al., the proportion of interval cancers after the negative screening test was $12 \%{ }^{13}$, in research by Zappa et al., the proportion was $11 \%^{21}$, in Netherlands $23 \%^{22}$ and in Basque Country (Spain), the proportion was $6.8 \%{ }^{23}$. Slovenian data can be compared with data, published in research by Zorzi et al..$^{13}$ and with data from Basque Country in Spain ${ }^{23}$, because the same type of immunochemical tests and the same cut-off value for positive test were used. Despite the fact that in Slovenia two stool samples are used in the screening programme, the proportion of interval cancers is similar to Zorzi et al. research. ${ }^{13}$

On six screen detected cancers, one case of interval cancer after negative faecal immunochemical test was detected in Slovenian screening programme, which is less than elsewhere. ${ }^{22}$

It has been reported that the screened population is at lower risk of colorectal cancer than the general population. ${ }^{20,24}$ This was associated with a healthier lifestyle, greater attention to symptoms and signs of possible diseases and higher participation rate in the screening programme. ${ }^{20,24} \mathrm{In}$ test sensitivity calculation it would be therefore inappropriate to use the general underlying incidence rates to estimate the expected cancers in the screened population (selection bias). ${ }^{18-20}$ That is why we used proportional incidence method to calculate test sensitivity. It includes proportion of screened persons, incidence of interval cancers detected after the negative screening test, incidence 
of colorectal cancers in the population prior to the introduction of the screening programme and incidence of non-responders' colorectal cancers.

The test sensitivity of the faecal immunochemical screening test was $88.45 \%$. Test sensitivity in other screening programmes rated between $77.0 \%$ and $87.0 \%{ }^{13,21,22,25}$ Proportional incidence method with the consideration of selection bias was used in Slovenia and in research by Zorzi et al. ${ }^{13}$ In the latter, test sensitivity was $79.3 \%$; taking into account that only one stool sample was used in a screening episode, while in Slovenia, two stool samples were tested. The age of target population was the same in both programmes. Despite the similar scope of the target population, Zorzi et al. ${ }^{13}$ reported more detected interval cancers. The use of two stool samples in our research might contributed to higher test sensitivity. Most likely, the proportion of cancers which could be detected as interval cancers, were already detected in the screening process, because of the positive second sample test result, which added 88 screen detected cancers, and this equals to $17.8 \%$ of all screen detected cancers.

The present data analysis on detected cancers has, similarly as researches in Italy, Spain, Scotland and Netherlands, confirmed that screen detected cancers are statistically more often detected in lower, more favourable stages (TNM stage I) compared to interval cancers or non-responders' detected cancers. In the latter, more cancers were detected in stages III and IV. 13,22,23,25,26 Promising finding that interval cancers are more frequently detected in stage I than non responders' cancers deserves further research.

As we expected, results of our study showed that there are significant differences between cancer sites in the colorectum according to detection mode. Significantly more screen detected and non-responders' cancers were detected in the left part of the colon, while more interval cancers were detected in the right part of the colon. This is in accordance with findings in researches by Portillo et al. ${ }^{23}$, Steele et al. ${ }^{26}$, Morris et al. ${ }^{27}$, and Gill et al..$^{28}$ In Italy ${ }^{13}$, there were no differences in the sites of interval cancers. In Netherlands' research, more cancers (interval, screen detected and nonresponders') were detected in the left part of the colon $^{22}$, and in Spanish research, interval cancers, compared to screen detected cancers, were more frequently detected in the rectum ${ }^{25}$. Differences in cancer site within the colorectum were observed across different studies. ${ }^{11,13,26,27}$ Possible explanation for this is that the screening test is being less effective at detecting right sided cancers in com- parison with left sided lesions. ${ }^{11,13,27}$ Haemoglobin from right sided colon lesions has longer time available to degrade when passing the colorectum and more false negative results may appear for right sided cancers. ${ }^{11,27}$

All three groups of cancer cases in our study (screen detected, interval and non-responders'), Table 1, did not differ significantly according to gender or age group; in general, cancers were more frequent among older people (aged 60+) and males. Similar results were found in Netherlands and in Spain. $22,23,25$ However, in two studies, where they used guaiac test (in Scotland and in Finland), proportion of interval cancers was higher in women compared to men. ${ }^{11,29}$

In order to estimate the influence of person's socio-economic status on cancer incidence, the income or person's education or the deprivation category of the neighbourhood in which this person lives is used. Socio-economic status was not an important factor for interval, non-responder or screen detected cancer in some studies. ${ }^{22,23,28}$ In research by Steele et al. ${ }^{26}$, significantly more interval and screen detected cancers were found in people living in less deprived surroundings compared to non-responders' detected cancers. This confirms the fact that persons with good socio-economic status are more responsively joining the screening programme. In our research, we used the educational attainment expressed in years of schooling as the socio-economic status indicator and it proved as significant for different mode of cancer detection. Persons with 10 or more years of schooling had significantly more interval and screen detected cancers. Impact of education on screen detected cancer group can be interpreted in the same way as impact of neighbourhood deprivation index of the study in Scotland. Among Slovenian non-responders cancer patients there is significantly more persons with less than 10 years of schooling.

In one fourth of interval cancer patients with negative quantitative screening test, the presence of haemoglobin was not detected at the time of two stool samples analysis (the value of haemoglobin was $0.0 \mathrm{ng} \mathrm{Hb} / \mathrm{mL}$ ). This confirms the fact that some cancers would be missed regardless of cut-off value for positive test result. Similar as in research by Barnett $\mathrm{KN}$ et al. ${ }^{30}$, we find that it is important to empower and inform people that in spite of the negative screening test, cancer can appear; therefore, they need to carefully monitor the symptoms, which indicate that diseases processes can be developing in the colon and rectum, which demand immediate visit to the general practitioner. ${ }^{30}$ Out of 57 
persons with interval cancer who used quantitative screening test, as many as $43(75.4 \%)$ had haemoglobin value under $50 \mathrm{ng} \mathrm{Hb} / \mathrm{mL}$ of buffer. Digby et $a l .{ }^{31}$ report similar findings. In their study, 23 (74.2\%) out of 31 persons with interval cancer had haemoglobin value under $50 \mathrm{ng} \mathrm{Hb} / \mathrm{mL}$ of buffer. The data of Slovenian and Scottish researches show that most persons with interval cancer had much lower haemoglobin value in stool samples, which were analysed prior to cancer diagnosis, as was set as cut-off value for positive screen test result. The cut-off value for positive screening test result in Slovenian research was the value of haemoglobin higher than $100 \mathrm{ng} \mathrm{Hb} / \mathrm{mL}$ of buffer in at least one of two stool samples, while in Digby et al. ${ }^{31}$, the cutoff value was set at $400 \mathrm{ng} \mathrm{Hb} / \mathrm{mL}$ of buffer.

As an advantage of our research, we should mention that in Slovenia we have population based national cancer registry with good quality data, from where we obtained the data on interval cancers and non-responders' cancers, as well as the cancer incidence prior to the introduction of screening. This enabled the calculation of test sensitivity following the proportional incidence method while considering the selection bias. Personal identification number enabled individual linkage of screening with cancer incidence data.

The limitation of screening registry at the time of the study is that it does not have legally guaranteed access to data on exclusion criteria in target population and it, therefore, has to rely on patients' self-reported data on colorectal diseases, which can sometimes be unreliable. It is possible that symptomatic persons who were already in diagnostic procedure, attended for screening. If colonoscopies after the positive immunochemical test in the screening were performed outside the screening programme, it depended on patients themselves if they forwarded the colonoscopy results to the screening registry. $7.8 \%$ of people with positive faecal immunochemical test did not undergo a colonoscopy inside the screening programme, but some of them have undergone a colonoscopy outside the screening programme. We could not estimate for how many of them we were not able to obtain the colonoscopy results with premalignant or non-malignant findings. From cancer registry only cancer cases are available.

\section{Conclusions}

The proportion of interval cancers after immunochemical faecal occult blood test in Slovenian colo- rectal cancer screening programme is similar to other programmes in Europe. Test sensitivity for immunochemical faecal test is among the highest when compared to similar programmes, which can be attributed to the analysis of two stool samples. Even the lowering of cut-off value for positive test result to the lowest noticeable value would not eliminate the interval cancers, because they are sometimes found in persons where there were no trace of faecal blood.

Important differences in stages and sites of detected cancers among interval, screen detected and non-responders' cancers are found. There are differences in socio-economic status of persons, in which cancer was detected as interval or screen detected, as well as in those, which were diagnosed with cancer without responding to the screening. In latter, there are characteristically more persons with lower education. Due to the more frequent diagnoses of cancer in higher stages among non-responders, there is a need for better awareness raising among the target population on the fact that participation in the screening programme enables early detection of cancer or its prevention.

\section{References}

1. Ferlay J, Soerjomataram I, Dikshit R, Eser S, Mathers C,Rebelo M. Cancer incidence and mortality worldwide: sources, methods and major patterns in GLOBOCAN 2012. Int J Cancer 2015; 136: E359-86. doi: 10.1002/ijc.29210

2. Zadnik V, Primic Zakelj M, Lokar K, Jarm K, Ivanus U, Zagar T. Cancer burden in Slovenia with the time trends analysis. Radiol Oncol 2017; 51: 47-55. doi: 10.1515/raon-2017-0008

3. Proposal for a Council Recommendation on Cancer Screening. Commission of the European Communities. 2003/0093(CNS). [cited 2018 Mar 15]. Available from: http://ec.europa.eu/health/ph_determinants/genetics/ documents/com_2003_0230_en.pdf

4. Mandel JS, Bond JH, Church TR, Snover DC, Bradley GM, Schuman LM, et al. Reducing mortality from colorectal cancer by screening for fecal occult blood. Minnesota Colon Cancer Control Study. N Engl J Med 1993; 328 1365-71. doi: 10.1056/NEJM199305133281901

5. Kronborg O, Fenger C, Olsen J, Jorgensen OD, Sondergaard O. Randomised study of screening for colorectal cancer with faecal-occult-blood test. Lancet 1996; 348: 1467-71. doi: 10.1016/S0140-6736(96)03430-7

6. Hardcastle JD, Chamberlain JO, Robinson MH, Moss SM, Amar SS, Balfour TW, et al. Randomised controlled trial of faecal-occult-blood screening for colorectal cancer. Lancet 1996; 348: 1472-7. doi: 10.1016/S01406736(96)03386-7

7. Segnan N, Patnick J, von Karsa L, editors. European Guidelines for Quality Assurance in Colorectal Cancer Screening and Diagnosis. First edition. European Commission. Luxembourg: Publications Office of the European Union; 2010 [cited 2018 Mar 15]. Available from: http://www.stopdarmkanker.be/BOEKJE/boekEU.pdf

8. Duffy MJ, van Rossum LG, van Turenhout ST, Malminiemi O, Sturgeon C Lamerz R, et al. Use of faecal markers in screening for colorectal neoplasia: a European group on tumor markers position paper. Int J Cancer 2011; 128: 3-11. doi: $10.1002 /$ ijc. 25654

9. Raffle A, Gray M. Screening: Evidence and Practice. Oxford: University Press; 2007. 
10. Sanduleanu S, le Clercq CM, Dekker E, Meijer GA, Rabeneck L, Rutter $\mathrm{MD}$, et al. Definition and taxonomy of interval colorectal cancers: a proposal for standardising nomenclature. Gut 2015; 64: 1257-67. doi: 10.1136/ gutjnl-2014-307992

11. Steele RJ, McClements P, Watling C, Libby G, Weller D, Brewster DH, et al. Interval cancers in a FOBT-based colorectal cancer population screening programme: implications for stage, gender and tumour site. Gut 2012; 6: 576-81. doi: 10.1136/gutjnl-2011-300535

12. Grazzini G, Visioli CB, Zorzi M, Ciatto S, Banovich F, Bonanomi AG, et al. Immunochemical faecal occult blood test: number of samples and positivity cutoff. What is the best strategy for colorectal cancer screening? Br J Cancer 2009; 100: 259-65. doi: 10.1038/sj.bjc.6604864

13. Zorzi M, Fedato C, Grazzini G, Stocco FC, Banovich F, Bortoli A, et al. High sensitivity of five colorectal screening programmes with faecal immunochemical test in the Veneto Region, Italy. Gut 2011; 60: 944-9. doi: 10.1136/ gut.2010.223982

14. Tepeš B, Stefanovič M, Bračko M, Frković Grazio S, Maučec Zakotnik J, Novak Mlakar D, et al. [Slovenian colorectal cancer screening programme SVIT - results of pilot phase.] [Slovenian]. Zdrav Vestn 2010; 79: 403-11.

15. Tepeš B, Bracko M, Novak Mlakar D, Stefanovic M, Stabuc B, Frkovic Grazio $\mathrm{S}$, et al. Results of the FIT-based National Colorectal Cancer Screening Program in Slovenia. J Clin Gastroenterol 2017; 51: e52-9. doi: 10.1097/ MCG.0000000000000662

16. Tepeš B, Kasesnik K, Novak Mlakar D, editos. [Slovenian guidelines of colorectal cancer screening programme SVIT: Slovenian guidelines ensure the quality of colorectal cancer screening.] [Slovenian]. First edition. Ljubljana: Nacionalni inštitut za javno zdravje; 2016.

17. International Classification of Diseases for Oncology, 3rd Edition. Geneva, Switzerland: World Health Organization; 2000.

18. Hakama M, Auvinen A, Day NE, Miller AB. Sensitivity in cancer screening. J Med Screen 2007; 14: 174-7. doi: 10.1258/096914107782912077

19. Malila N, Oivanen T, Malminiemi O, Hakama M. Test, episode, and programme sensitivities of screening for colorectal cancer as a public health policy in Finland: experimental design. BMJ 2008; 337: a2261. doi: 10.1136/ bmj.a2261

20. GISCoR Working Group, Zorzi M (Coordinator). Detection of the interval cancers and estimate of the sensitivity of colorectal cancer screening programmes. Working report. Epidemiol Prev 2013; 37 (2-3 Suppl 1) [cited 2018 Mar 15]. Available from: http://www.epiprev.it/materiali/2013/EP2-3/ S1_GISCOR/GISCOR_2013_Eng_def.pdf

21. Zappa M, Castiglione G, Paci E, Grazzini G, Rubeca T, Turco P, et al. Measuring interval cancers in population-based screening using different assays of fecal occult blood testing: the District of Florence experience. Int J Cancer 2001; 92: 151-4. doi: 10.1002/1097-0215(200102)9999:9999<::AIDIJC1149>3.0.CO;2-6

22. van der Vlugt M, Grobbee EJ, Bossuyt PMM, Bos A, Bongers E, Spijker W, et al. Interval Colorectal Cancer Incidence Among Subjects Undergoing Multiple Rounds of Fecal Immunochemical Testing. Gastroenterology 2017; 153: 439-47. doi: 10.1053/j.gastro.2017.05.004

23. Portillo I, Arana-Arri E, Idigoras I, Bilbao I, Martínez-Indart L, World LB, et al. Colorectal and interval cancers of the Colorectal Cancer Screening Program in the Basque Country (Spain). J Gastroenterol 2017; 23: 2731-42. doi: 10.3748/wjg.v23.i15.2731

24. Senore C, Armaroli P, Silvani M, Andreoni B, Bisanti L, Marai L, et al. Comparing different strategies for colorectal cancer screening in Italy: predictors of patients' participation. Am J Gastroenterol 2010; 105: 188-98. doi: 10.1038/ajg.2009.583

25. Garcia $M$, Domènech $X$, Vidal $C$, Torné $E$, Milà $N$, Binefa $G$, et al. Interval Cancers in a Population-Based Screening Program for Colorectal Cancer in Catalonia, Spain. Gastroenterol Res Pract 2015; 2015: 672410. Published online 2015 Feb 24. doi: 10.1155/2015/672410

26. Steele RJ, Stanners G, Lang J, Brewster DH, Carey FA, Fraser CG. Interval cancers in a national colorectal cancer screening programme. United European Gastroenterol J 2016; 4: 587-94. doi: 10.1177/2050640615624294

27. Morris EJA, Whitehouse LE, Farrell T, Nickerson C, Thomas JD, Quirke $P$, et al. A retrospective observational study examining the characteristics and outcomes of tumours diagnosed within and without of the English NHS Bowel Cancer Screening Programme. Br J Cancer 2012; 107: 757-64. doi: 10.1038/bjc.2012.331
28. Gill MD, Bramble MG, Rees CJ, Lee TJ, Bradburn DM, Mills SJ. Comparison of screen-detected and interval colorectal cancers in the Bowel Cancer Screening Programme. Br J Cancer 2012; 107: 417-21. doi: 10.1038/ bjc. 2012.305

29. Paimela H, Malila N, Palva T, Hakulinen T, Vertio H, Järvinen H. Early detection of colorectal cancer with faecal occult blood test screening. Br J Surg 2010; 97: 1567-71. doi: 10.1002/bjs.7150

30. Barnett KN, Weller D, Smith S, Steele RJ, Vedsted P, Orbell S, et al. The contribution of a negative colorectal screening test result to symptom appraisal and help-seeking behaviour among patients subsequently diagnosed with an interval colorectal cancer. Health Expect 2018. doi: 10.1111/hex.12672

31. Digby J, Fraser CG, Carey FA, Lang J, Stanners G, Steele RJ. Interval cancers using a quantitative faecal immunochemical test (FIT) for haemoglobin when colonoscopy capacity is limited. J Med Screen 2016; 23: 130-4. doi: 10.1177/0969141315609634 